\title{
A KECK SURVEY OF GRAVITATIONAL LENS SYSTEMS. I. SPECTROSCOPY OF SBS 0909+532, HST 1411+5211, AND CLASS B2319+051
}

\author{
L. M. LUBIN ${ }^{1}$ \\ Palomar Observatory, California Institute of Technology, Mail Stop 105-24, Pasadena, CA 91125;1ml@astro.caltech.edu \\ C. D. FASSNACHT \\ National Radio Astronomy Observatory, P.O. Box O, Socorro, NM 87801; cfassnac@aoc.nrao.edu \\ A. C. S. ReADHEAD \\ Palomar Observatory, California Institute of Technology, Mail Stop 105-24, Pasadena, CA 91125; acr@astro.caltech.edu \\ R. D. BLANDFORD \\ Theoretical Astrophysics, California Institute of Technology, Mail Stop 130-33, Pasadena, CA 91125; rdb@tapir.caltech.edu \\ AND \\ T. Kundić \\ Renaissance Technologies, 600 Route 25A, East Setauket, NY 11733 \\ Received 1999 September 22; accepted 1999 October 25
}

\begin{abstract}
We present new results from a continuing Keck program designed to study gravitational lens systems. We have obtained redshifts for three lens systems, SBS 0909+532, HST 1411+5211, and CLASS B2319+051. For all of these systems, either the source or lens redshift (or both) has been previously unidentified. Our observations provide some of these missing redshifts. We find $\left(z_{l}, z_{s}\right)=(0.830,1.377)$ for SBS $0909+532 ;\left(z_{l}, z_{s}\right)=(0.465,2.811)$ for HST $1411+5211$, although the source redshift is still tentative; and $\left(z_{l, 1}, z_{l, 2}\right)=(0.624,0.588)$ for the two lensing galaxies in CLASS B2319+051. The background radio source in B2319+051 has not been detected optically; its redshift is, therefore, still unknown. We find that the spectral features of the central lensing galaxy in all three systems are typical of an early-type galaxy. The observed image splittings in SBS $0909+532$ and HST $1411+5211$ imply that the masses within the Einstein ring radii of the lensing galaxies are $1.4 \times 10^{11}$ and $2.0 \times 10^{11} h^{-1} M_{\odot}$, respectively. The resulting $B$-band mass-to-light ratio $(M / L)$ for HST $1411+5211$ is $41.3 \pm 1.2 h(M / L)_{\odot}$, a factor of $\sim 5$ times higher than the average early-type lensing galaxy. This large mass-to-light ratio is almost certainly the result of the additional mass contribution from the cluster CL 3C 295 at $z=0.46$. For the lensing galaxy in SBS $0909+532$, we measure $(M / L)_{B}=4_{-3}^{+11} h(M / L)_{\odot}$, where the large errors are the result of significant uncertainty in the galaxy luminosity. While we cannot measure directly the mass-tolight ratio of the lensing galaxy in $\mathrm{B} 2319+051$, we estimate that $(M / L)_{B}$ is between 3-7 $h(M / L)_{\odot}$.

Key words: distance scale - galaxies: distances and redshifts - gravitational lensing -

quasars: individual (CLASS 2319+051, HST 1411+5211, SBS 0909+532)
\end{abstract}

\section{INTRODUCTION}

Gravitational lensing has proved to be an invaluable astrophysical tool for constraining the cosmological parameters $H_{0}$ (Kundić et al. 1997c; Schechter et al. 1997; Lovell et al. 1998; Biggs et al. 1999; Fassnacht et al. 1999) and $\Lambda$ (Falco, Kochanek, \& Muñoz 1998; Helbig et al. 1999). In addition, a unique contribution of gravitational lensing to extragalactic astronomy lies in its capacity to measure directly the masses of the lensing objects. Consequently, it can be used to study galaxy structure and its evolution with redshift (e.g., Keeton, Kochanek, \& Falco 1998). The advent of high spatial resolution imaging with Hubble Space Telescope $(H S T)$ and faint-object spectroscopy with the Keck 10 $\mathrm{m}$ telescopes have opened new possibilities in the field (e.g., Kundić et al. 1997a, 1997b; Fassnacht \& Cohen 1998). Systems with compact configurations and faint components can now be studied, increasing the size and completeness of statistical samples of lenses. Specifically, a detailed study of a large number of gravitational lens systems can be used (1)

${ }^{1}$ Hubble Fellow. to identify simple lens systems for the measurement of $H_{0}$, (2) to measure the mass-to-light ratio $(M / L)$ of the lensing galaxies, (3) to compare the dark matter with the stellar light distribution of the lens galaxies, and (4) to probe the interstellar medium in the lensing galaxies. Nearly all of these goals depend critically on accurate redshift determinations for the background sources and the lensing galaxies.

In light of this, we have begun a coordinated program to use the Low Resolution Imaging Spectrograph (LRIS; Oke et al. 1995) on the Keck II Telescope to measure spectroscopic redshifts for all lens systems where either the source or lens redshift is currently unavailable. We have drawn our sources from the sample of the CfA-Arizona Space Telescope Lens Survey (CASTLES) of gravitational lenses. The CASTLES team has compiled a list of all known confirmed or candidate gravitational lens systems with angular separations smaller than $10^{\prime \prime}$. These systems were originally identified by a variety of methods and by many different groups. The specific goal of CASTLES is the construction of a complete three-band $(V, I$, and $H$ ) photometric survey of this sample. CASTLES uses existing HST images when available. Otherwise, they have supplemented the archival 
data with new Wide Field Planetary Camera 2 (WFPC2) and/or NICMOS imaging. ${ }^{2}$

In addition, we have prepublication access to new gravitational lens candidates discovered in the Cosmic Lens All-Sky Survey (CLASS). CLASS is being conducted at radio wavelengths with the Very Large Array (VLA) and consists of observations of $\sim 12,000$ flat-spectrum radio sources to search for gravitational lens candidates. The first three phases of this survey have confirmed 12 new lenses and found $\sim 10$ additional candidates (Myers et al. 2000). As part of CLASS follow-up observations, many of these lenses have been imaged in two or three bands with HST (Jackson et al. 1998a, 1998b; Koopmans, de Bruyn, \& Jackson 1998; Koopmans et al. 1999; Sykes et al. 1998; Fassnacht et al. 1999; Xanthopoulos et al. 1998). At this time, eight of the 12 confirmed lenses from CLASS are included in CASTLES.

Earlier results from the first phases of this Keck survey have already been published (Kundic et al. 1997a, 1997b; Fassnacht \& Cohen 1998). In this paper, we present spectra of three lens systems with missing redshifts: SBS 0909+532, HST $1411+5211$, and CLASS B2319+051. Unless otherwise noted, we use $H_{0}=100 h \mathrm{~km} \mathrm{~s}^{-1} \mathrm{Mpc}^{-1}, \Omega_{m}=0.2$, and $\Omega_{\Lambda}=0.0$.

\section{TARGETS}

Below we present some relevant information on the previous observations of the three lens systems that are the subject of this paper.

\section{1. $S B S 0909+532$}

SBS $0909+532$ was first discovered as a quasar by Stepanyan et al. (1991) and later identified in the Hamburg-CfA Bright Quasar Survey (Engels et al. 1998). Kochanek, Falco, $\&$ Schild (1995) believed that this quasar was a good candidate for gravitational lensing because of its redshift $(z=1.377)$ and its bright optical magnitude $(B=17.0)$. Kochanek et al. (1997) first resolved this source into a close pair that was separated by $\Delta \theta=1$ ".11 and had a flux ratio of $R_{\mathrm{B}}-R_{\mathrm{A}}=0.58 \mathrm{mag}$. These observations suggested that this system was indeed a gravitational lens. Oscoz et al. (1997) confirmed this hypothesis with spectra of the two components taken at the William Herschel Telescope. The spectra showed that components A and B were quasars at the same redshift and had identical spectra. Oscoz et al. (1997) also detected the $\mathrm{Mg}$ II $\lambda 22796,2803$ doublet in absorption at the same redshift $(z=0.83)$ in both components. They argued that these absorption features were associated with the photometrically unidentified lensing galaxy. Optical and infrared $H S T$ imaging indicate that the lensing galaxy has a large effective radius $\left(r_{\text {eff }}=1\right.$ 1.58 \pm 0 ".90) and a correspondingly low surface brightness. It has a total magnitude of $H=16.75 \pm 0.74$ and a color of $I-H=2.28 \pm 1.01$ within an aperture of diameter $1^{\prime \prime} .7$ (Lehár et al. 2000). The large uncertainties are a result of the difficulty in subtracting the close pair of quasar images (see Fig. 1 of Lehár et al. 2000). Our observations confirm that the lensing galaxy is at the same redshift as the $\mathrm{Mg}$ II absorbers.

\footnotetext{
${ }^{2}$ See http://cfa-www.harvard.edu/castles.
}

\section{2. $H S T 1411+5211$}

HST $1411+5211$ is a quadruple lens that was discovered by Fischer, Schade, \& Barrientos (1998) in archival WFPC2 images taken of the cluster CL 3C 294 (CL 1409+5226) with the F702W filter. The maximum image separation is 2 ".28. The intensities of the four components are reasonably similar; the F702W AB magnitudes correspond to (A, B, C, $\mathrm{D})=(24.96,25.95,24.92,25.00)$. The primary lensing galaxy is clearly observed in the $H S T$ images with a total $\mathrm{AB}$ magnitude of F702W $=20.78 \pm 0.05$. It has the appearance of a morphologically normal elliptical galaxy with a measured half-light radius of $r_{1 / 2}=00^{\prime \prime} 61 \pm 0.03$ and an ellipticity of $\epsilon=0.27 \pm 0.03$. The lensing galaxy is located only 52 ". (or $195 h^{-1} \mathrm{kpc}$ ) from the center of the massive cluster CL 3C 295 at $z=0.46$ (Butcher \& Oemler 1978). Although this cluster was the subject of an extensive spectroscopic survey by Dressler \& Gunn (1992), there is no measured redshift for the lensing galaxy (identified as galaxy 162 in Table 6 of Dressler \& Gunn 1992); however, a photometric redshift of $z=0.598 \pm 0.11$ based on narrowband imaging has been measured (Thimm et al. 1994). Fischer et al. (1998) argued that this photometric redshift was suspect. First, the photometric redshift had the largest quoted uncertainty of all the observed galaxies (over 2 times larger than the average). Second, Thimm et al. (1994) classified this galaxy as Scd based on their measurement of the spectral energy distribution. The high angular resolution HST imaging clearly indicates that this galaxy is an early-type, not a latetype, galaxy. In this paper, we convincingly show that the photometric redshift of Thimm et al. (1994) is incorrect.

\subsection{CLASS B2319+051}

B2319+051 is a doubly imaged gravitational lens system newly discovered by CLASS (Marlow et al. 2000). Radio images taken with the VLA and the Multiple-Element Radio-Linked Interferometer Network (MERLIN) show two compact components aligned in a north-south orientation with a separation of 1".36 and a flux density ratio of 5.7:1. High-resolution radio imaging with the Very Long Baseline Array resolve each component into two subcomponents with a separation of 0".021 for A and 0"0075 for $B$. The orientation and morphology of this configuration are consistent with the lensing hypothesis. Images of this system taken with NICMOS do not show any infrared counterparts to the radio components; however, they do reveal two lensing galaxies (Marlow et al. 2000). G1 is a large, elliptical-like galaxy that is associated with the position of the two radio components; hence, it is the primary lensing galaxy. G2 is an extended, irregular galaxy that shows two clear emission peaks (G2a and G2b) and is separated from G1 by G1-G2b=3.516 (see Fig. 9 of Marlow et al. 2000). This galaxy is the source of an external shear as modeled by Marlow et al. (2000). The integrated magnitudes of $\mathrm{G} 1$ and $\mathrm{G} 2$ are $\mathrm{F} 160 \mathrm{~W}=18.2$ and 19.1, respectively.

\section{OBSERVATIONS}

All of the observations were performed with LRIS (Oke et al. 1995) on the Keck II Telescope. For the spectroscopic observations, we have used the instrument in long-slit mode with the 300 grooves $\mathrm{mm}^{-1}$ grating, which provides a spectral resolution of $2.44 \AA$ pixel $^{-1}$. The long slit was aligned along the axis defined by the two images of the background 
TABLE 1

OBSERVATIONS

\begin{tabular}{|c|c|c|c|c|c|}
\hline System & Date & $\begin{array}{c}t_{\exp } \\
(\mathrm{s})\end{array}$ & $\begin{array}{l}\text { Slit Width } \\
\text { (arcsec) }\end{array}$ & $\begin{array}{l}\text { P.A. } \\
\text { (deg) }\end{array}$ & $\begin{array}{c}\text { Coverage } \\
\text { (A) }\end{array}$ \\
\hline SBS $0909+532$. & 1997 Dec 27 & 1200 & 1.0 & 115.2 & $3802-8783$ \\
\hline HST $1411+5211 \ldots$ & 1998 Jun 29 & 5400 & 0.7 & 100.6 & $3855-8836$ \\
\hline CLASS B2319+051 G1 ..... & 1998 Aug 1 & 3600 & 1.0 & 0.0 & $4009-8991$ \\
\hline CLASS B2319+051 G2..... & 1999 Jul 15 & 1500 & 0.7 & 45.0 & 4131-9132 \\
\hline
\end{tabular}

source for both SBS $0909+532$ and CLASS B2319+051. Note that the latter position covers the primary lensing galaxy G1 in the B2319+051 system but does not cover G2. For galaxy G2, the long slit was placed along the axis defined by its two components, G2a and G2b (see $\S 2.3$ ). For HST 1411+5211, the long slit was aligned along the axis defined by images $\mathrm{A}$ and $\mathrm{C}$ of the background source (see Fischer et al. 1998). Except for galaxy G2 of B2319+051, where only one exposure was taken, two exposures of equal duration were taken for each object. The specific details of these observations are listed in Table 1 . In addition, we have obtained $R$ images of CLASS B2319+051 using LRIS in imaging mode. These data are the only optical imaging available on this source. The total exposure time for these observations is $1200 \mathrm{~s}$.

In all cases, the data were reduced using standard IRAF ${ }^{3}$ routines. The bias levels were estimated from the overscan region on each chip. For the imaging data, a flat field was constructed from dome flats taken in the beginning of each night. For the spectroscopic observations, flat-fielding and wavelength calibration were performed using internal flat-

${ }^{3}$ IRAF is distributed by the National Optical Astronomy Observatories, which are operated by the Association of Universities for Research in Astronomy, Inc., under cooperative agreement with the National Science Foundation. field and arc-lamp exposures that were taken after each science exposure. Observations of the Oke (1990) spectrophotometric standard stars Feige 34, G138-31, BD $+33^{\circ} 2642$, and Feige 110 were used to remove the response function of the chip. The individual spectra for each object were weighted by the squares of their signal-to-noise ratios and combined.

\section{RESULTS}

The final spectra are shown in Figures 1-3, 5, and 6. The lines used to identify the redshifts of the lensing galaxies and the background sources are given in Table 2. The redshift uncertainties (see Table 3 ) have been estimated by taking the rms scatter in the redshifts calculated from the individual spectral lines. We present a more detailed discussion of the individual systems below.

\section{1. $S B S 0909+532$}

The spatial projection of the spectra from the SBS $0909+532$ system shows a double peak, with the subpeaks separated by approximately 5 pixels, or 1 ".1. This separation matches the 1".107 quasar image separation measured by Kochanek et al. (1997). The spectrum shown in Figure 1 was extracted using an aperture of 2 pixels placed in the trough between the subpeaks of emission in order to maximize the fractional contribution of the lensing galaxy. The

TABLE 2

DeteCted SPECTRAL LiNeS

\begin{tabular}{|c|c|c|c|c|c|c|c|}
\hline \multirow[b]{2}{*}{ IoN } & \multirow{2}{*}{$\begin{array}{l}\lambda_{0} \\
(\AA)\end{array}$} & \multicolumn{2}{|c|}{ SBS $0909+532$} & \multicolumn{2}{|c|}{ HST $1411+5211$} & \multicolumn{2}{|c|}{ CLASS B2319+051 } \\
\hline & & Source & Lens & Source & Lens & Lens 1 & Lens 2 \\
\hline $\operatorname{Ly} \alpha \ldots \ldots \ldots$ & 1216 & $\cdots$ & $\ldots$ & 4634 & $\ldots$ & $\ldots$ & $\ldots$ \\
\hline He II ......... & 1641 & 3901 & $\ldots$ & $\ldots$ & $\ldots$ & $\ldots$ & $\ldots$ \\
\hline $\mathrm{O} \mathrm{III}] \ldots \ldots \ldots$ & 1663 & 3953 & $\ldots$ & $\ldots$ & $\ldots$ & $\ldots$ & $\ldots$ \\
\hline $\mathrm{C}$ III] $\ldots \ldots \ldots$ & 1909 & 4538 & $\ldots$ & $\ldots$ & $\ldots$ & $\ldots$ & $\ldots$ \\
\hline $\mathrm{C}$ II $] \ldots \ldots \ldots$ & 2326 & 5529 & $\ldots$ & $\ldots$ & $\ldots$ & $\ldots$ & $\ldots$ \\
\hline \multirow[t]{3}{*}{$\mathrm{Fe}$ II ......... } & 2382 & $\ldots$ & 4359 & $\ldots$ & $\ldots$ & $\ldots$ & $\ldots$ \\
\hline & 2586 & $\ldots$ & 4732 & $\ldots$ & $\ldots$ & $\ldots$ & $\ldots$ \\
\hline & 2599 & $\ldots$ & 4756 & $\ldots$ & $\ldots$ & $\ldots$ & $\ldots$ \\
\hline $\operatorname{Mg}$ II $\ldots \ldots \ldots$ & 2796 & 6646 & 5117 & $\ldots$ & $\ldots$ & $\ldots$ & $\ldots$ \\
\hline $\operatorname{Mg}_{I} \ldots \ldots \ldots$ & 2853 & $\ldots$ & 5221 & $\ldots$ & $\ldots$ & $\ldots$ & $\ldots$ \\
\hline $\mathrm{O}$ III $\ldots . . . .$. & 3133 & 7447 & $\ldots$ & $\ldots$ & $\ldots$ & $\ldots$ & $\ldots$ \\
\hline \multirow[t]{2}{*}[\mathrm{Ne}\mathrm{v}]{$\ldots \ldots$} & 3346 & 7953 & $\ldots$ & $\ldots$ & $\ldots$ & $\ldots$ & $\ldots$ \\
\hline & 3427 & 8146 & $\ldots$ & $\ldots$ & $\ldots$ & $\ldots$ & $\ldots$ \\
\hline$[\mathrm{O} \mathrm{II}] \ldots \ldots .$. & 3727 & $\ldots$ & $\ldots$ & $\ldots$ & $\ldots$ & 6053 & 5921 \\
\hline $\mathrm{Ca}$ II $\mathrm{K} . . . .$. & 3934 & $\ldots$ & 7199 & $\ldots$ & 5758 & 6388 & 6250 \\
\hline $\mathrm{Ca}$ II $\mathrm{H} . . . .$. & 3968 & $\ldots$ & 7261 & $\ldots$ & 5814 & 6422 & 6308 \\
\hline $\mathrm{H} \delta \ldots \ldots \ldots$ & 4102 & $\ldots$ & $\ldots$ & $\ldots$ & 6013 & 6661 & 6511 \\
\hline $\mathrm{H} \gamma \ldots \ldots \ldots$ & 4341 & $\ldots$ & $\ldots$ & $\ldots$ & $\ldots$ & 7046 & $\ldots$ \\
\hline $\mathrm{H} \beta \ldots \ldots \ldots$ & 4841 & $\ldots$ & $\ldots$ & $\ldots$ & 7125 & $\ldots$ & 7729 \\
\hline
\end{tabular}

NoTE.- Observed wavelengths are in angstrom units. 
TABLE 3

LeNS SySTEM PARAMETers

\begin{tabular}{|c|c|c|c|c|c|c|c|}
\hline System & $z_{l}$ & $z_{s}$ & $\begin{array}{c}D_{l} \\
\left(h^{-1} \mathrm{Mpc}\right)\end{array}$ & $\begin{array}{c}D_{s} \\
\left(h^{-1} \mathrm{Mpc}\right)\end{array}$ & $\begin{array}{c}D_{l s} \\
\left(h^{-1} \mathrm{Mpc}\right)\end{array}$ & $\begin{array}{c}M_{\mathrm{E}} \\
\left(10^{11} h^{-1} M_{\odot}\right)\end{array}$ & $\begin{array}{c}(M / L)_{B} \\
{\left[h(M / L)_{\odot}\right]}\end{array}$ \\
\hline SBS $0909+532 \ldots \ldots \ldots \ldots$ & $0.8302 \pm 0.0001$ & $1.3764 \pm 0.0003$ & $999 \pm 0.02$ & $1129 \pm 0.01$ & $301 \pm 0.09$ & $1.42 \pm 0.03$ & $4_{-3}^{+11}$ \\
\hline HST $1411+5211 \ldots \ldots \ldots$ & $0.4641 \pm 0.0001$ & $2.811 \pm 0.005$ & $778 \pm 0.07$ & $1160 \pm 0.48$ & $730 \pm 0.13$ & $1.98 \pm 0.02$ & $41.3 \pm 1.2$ \\
\hline CLASS B2319+051 ..... & $0.6238 \pm 0.0001$ & $\ldots$ & $896 \pm 0.04$ & $\ldots$ & $\ldots$ & $\ldots$ & $\ldots$ \\
\hline
\end{tabular}

final spectrum is still dominated by light from the background source, a quasar at $z_{s}=1.377$ with broad C III] and Mg II emission lines (as seen in Oscoz et al. 1997). However, it is possible to see features from the lensing galaxy, including the $\mathrm{Ca}$ II $\mathrm{H}$ and $\mathrm{K}$ doublet, which establishes the lens redshift as $z_{l}=0.830$. The features identified with the lensing galaxy are typical of an early-type galaxy. For a nonevolving elliptical galaxy at the lens redshift, we expect an optical-infrared color of $I-H \sim 2$ (Poggianti 1997). Consequently, the observed value $I-H=2.28 \pm 1.01$ (Lehár et al. 2000) provides additional support for an earlytype classification of the lensing galaxy.

\section{2. $H S T 1411+5211$}

The spectrum of lens system HST $1411+5211$ shows two distinct traces, a bright central source that is separated by approximately 5 pixels, or 1 ". 1 , from a significantly fainter one. The two traces correspond to the lensing galaxy and the background source, respectively, as the separation is exactly that expected from the high angular resolution $H S T$ imaging (Fischer et al. 1998). From these spectra, we have obtained source and lens redshifts of $z_{l}=0.465$ and $z_{s}=$ 2.811 , respectively. In the spectrum of the lensing galaxy, the strong $4000 \AA$ break, the small equivalent-width Balmer absorption lines, and the lack of [O II] emission indicate that little star formation is occurring (see Fig. 2). The spectral features are consistent with the fact that this galaxy appears as a morphologically normal elliptical. The measured redshift proves that the lensing galaxy is a member of the cluster CL 3C 295.

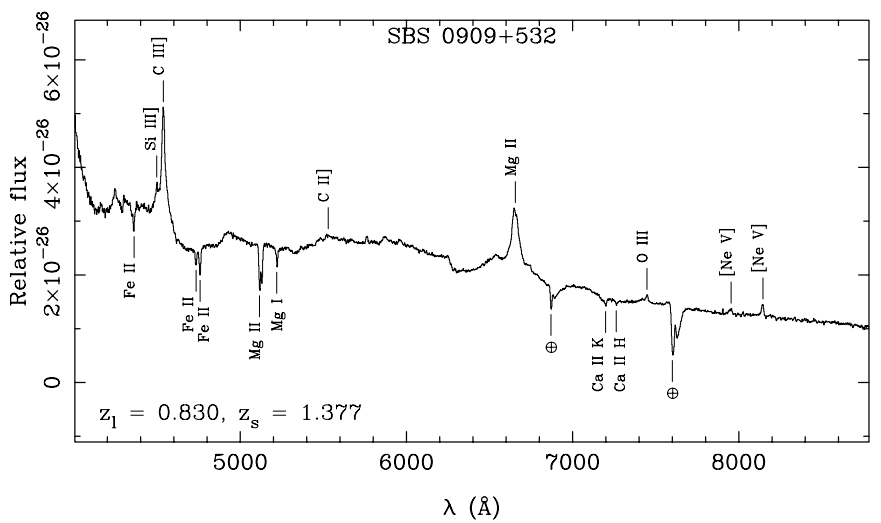

FIG. 1.-LRIS spectrum of the SBS $0909+532$ system. Flux calibration using the spectrophotometric standard Feige 34 has been performed. The vertical axis has been converted from $F_{\lambda}$ to $F_{v}$ in order to emphasize the absorption features associated with the lensing galaxy. Spectral lines from both the lensing galaxy $\left(z_{l}=0.830\right)$ and the background source $\left(z_{s}=1.377\right)$ are seen in the spectrum. All marked nonterrestrial absorption lines are due to the lensing galaxy, while all marked emission lines are due to the background source. The previously unmeasured lensing redshift is determined from the $\mathrm{Ca}$ II $\mathrm{H}$ and $\mathrm{K}$ features.
The background source shows a modest emission line at an observed wavelength of $4634 \AA$ (Fig. 3). This line is much more obvious in the two-dimensional, sky-subtracted spectrum than in the one-dimensional spectrum (see Fig. 4). There are only two plausible interpretations of this emission line, as all other choices would require the presence of other, stronger emission lines. First, the line could be $[\mathrm{O}$ II $] \lambda 3273$ at $z_{s}=0.243$. We would then expect to see comparably strong [O III] $\lambda \lambda 5007,4959$ at $6164,6224 \AA$ or $\mathrm{H} \beta \lambda 4861$ at

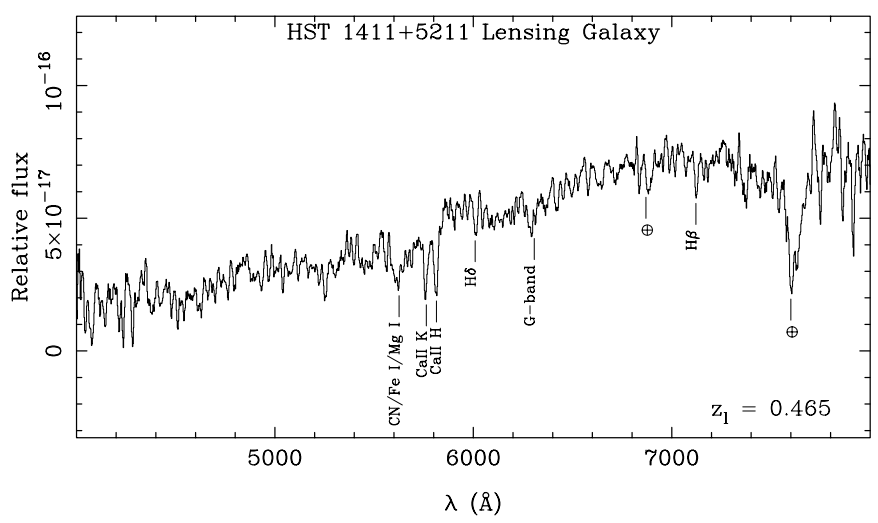

FIG. 2.-LRIS spectrum of the lensing galaxy in HST $1411+5211$. Flux calibration using the spectrophotometric standard G138-31 has been performed. The spectrum has been smoothed with a box car of size $12 \AA$. The previously unmeasured lensing redshift of $z_{l}=0.465$ is determined from the $\mathrm{Ca}$ II $\mathrm{H}$ and $\mathrm{K}, \mathrm{H} \delta$, and $\mathrm{H} \beta$ features.

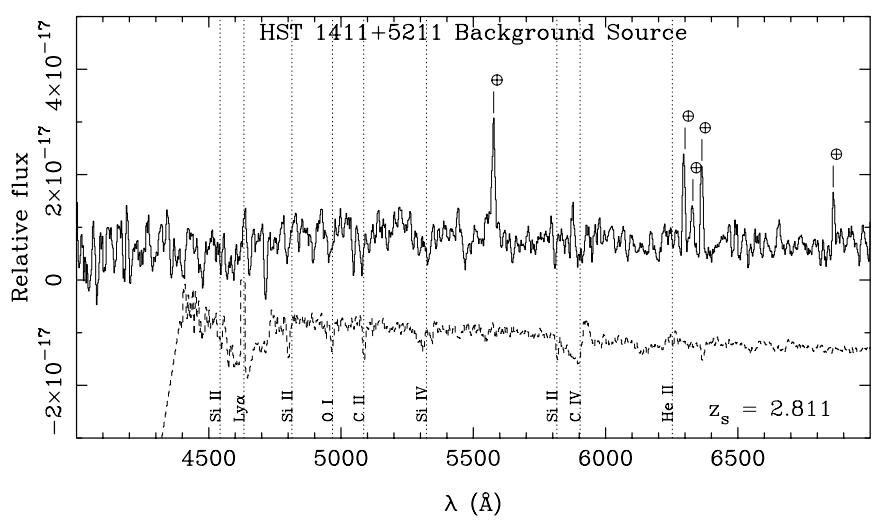

FIG. 3.-LRIS spectrum of the background source in HST $1411+5211$. Flux calibration using the spectrophotometric standard G138-31 has been performed. The spectrum has been smoothed with a box car of size $12 \AA$. The previously unmeasured source redshift of $z_{s}=2.811$ is determined from the Ly $\alpha$ line and several observed absorption features. Below this spectrum we plot a scaled spectrum of the nearby starburst galaxy NGC 4214 (Leitherer et al. 1996). The position of several stellar and interstellar features that are routinely observed in both nearby and distant starforming galaxies are indicated with vertical lines. 


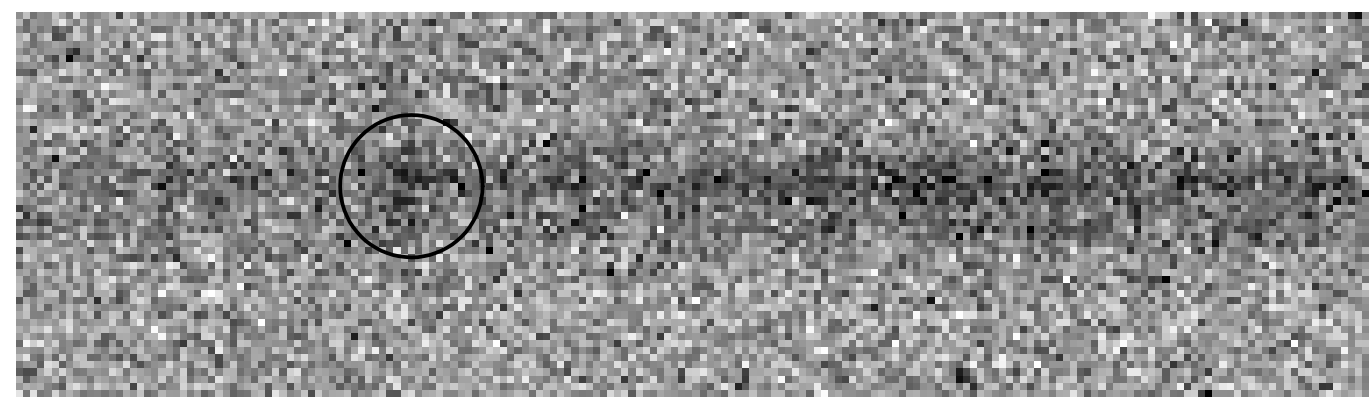

FIG. 4.-Two-dimensional, sky-subtracted spectrum of HST $1411+5211$. The brighter, upper trace belongs to the lensing galaxy. The fainter, bottom trace belongs to the source. The dispersion axis ranges from 4500 to $4965 \AA$, and the spatial axis covers $111^{\prime \prime} 8$. Emission from the Ly $\alpha$ line at the observed wavelength of $4643 \AA$ is circled. Note that the Ly $\alpha$ emission appears significantly extended.

$6042 \AA$ A. None of these lines are seen in the data, although the spectrum is much weaker at these wavelengths. This identification would also imply that the emission is not coming from the background source, but rather from some unrelated foreground object. Because of the lack of other emission lines and the exact coincidence with the position of the background source, we believe the only reasonable explanation for this line is $\operatorname{Ly} \alpha \lambda 1215.7$ at $z_{s}=2.811$. The appearance of this spectrum is similar to other known starforming galaxies at comparable redshifts with absorption features that include, e.g., Si II and C IV (Steidel et al. 1996a, 1996b). In addition, there is a continuum break blueward of this line with a drop amplitude (Oke \& Korycansky 1982) of $D_{A}=0.25 \pm 0.05$. This decrement is due to absorption by intervening hydrogen and is consistent with that found in the spectra of other high-redshift objects (see, e.g., Oke \& Korycansky 1982; Kennefick, Djorgovski, \& de Carvalho 1995). Because of the low signal-to-noise ratio in this spectrum, we still regard this redshift measurement as tentative. We are planning to reobserve this object during the next observing season.

In addition to the lens system, we have also obtained spectra of two galaxies that happened to lie on the long slit during the observations of the gravitational lens system. They are identified as galaxies 158 and 165 in the cluster field CL 3C 295 (see Table 6 of Dressler \& Gunn 1992). Dressler \& Gunn (1992) list their total $r$ magnitudes as 20.13 and 22.56, respectively. The redshift of each galaxy was previously unknown. Based on our spectra, we find a redshift of $z=0.451$ for both galaxies (Fig. 5), indicating that the galaxies are cluster members. Each spectrum shows the classic $\mathrm{K}$ star absorption features of $\mathrm{Ca}$ II $\mathrm{H} \& \mathrm{~K}$ that are typical of an early-type galaxy. In addition, they show a series of strong Balmer absorption lines, including $\mathrm{H} \theta, \mathrm{H} \eta$, $\mathrm{H} \zeta, \mathrm{H} \delta, \mathrm{H} \gamma$, and $\mathrm{H} \beta$, which suggests that these galaxies are " $\mathrm{K}+\mathrm{A}$ " (or more commonly known as " $\mathrm{E}+\mathrm{A}$ ") galaxies (Dressler \& Gunn 1983, 1992; Zabludoff et al. 1996). These spectral features imply that these galaxies have experienced a brief starburst within the last 1-2 Gyr.

\subsection{CLASS B2319+051}

We have obtained spectra of the two lensing galaxies, G1 and G2, in B2319+051 (see Fig. 6). No optical emission associated with the background radio source has been detected; thus, the source redshift is still unknown. The redshifts of the two lensing galaxies are $\left(z_{l, 1}, z_{l, 2}\right)=$ $(0.624,0.588)$. As the redshifts indicate, $\mathrm{G} 2$ is not a companion galaxy to the primary lensing galaxy G1. Rather, they are just a chance superposition along the line of sight. The spectrum of G1 is consistent with its morphological identification as an early-type galaxy in the high angular resolution NICMOS image (Marlow et al. 2000). It has a strong 4000 $\AA$ break and small equivalent-width Balmer absorption lines. It does, however, show some indication of current star formation with a modest [O $\mathrm{II}]$ line (equivalent width of 9 $\AA$ A). Galaxy G2 is clearly more active as it has much stronger [O II] emission (equivalent width of $22 \AA$ ) and a less welldefined $4000 \AA$ break. In addition, the spectrum shows a series of strong Balmer absorption features that indicates a
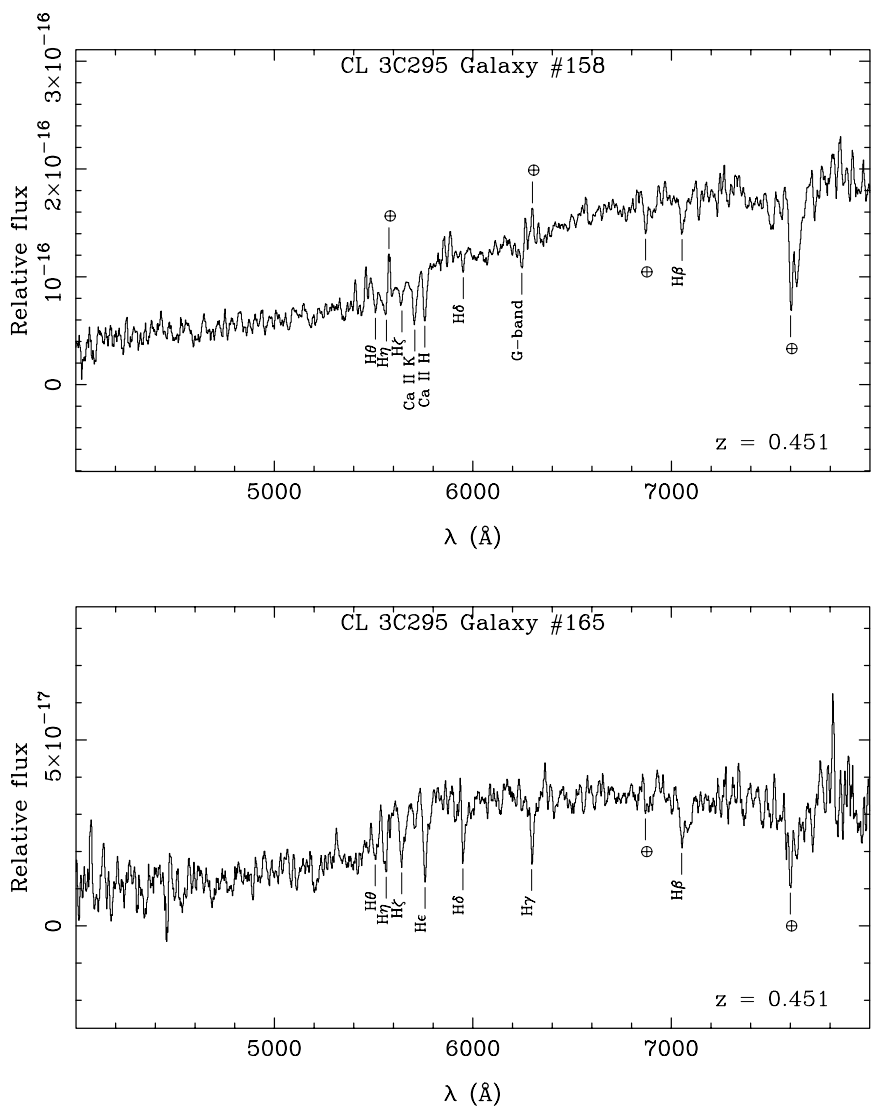

FIG. 5.-LRIS spectra of two cluster members in CL 3C 295 (CL 1409+5226). Galaxy 158 (top) and galaxy 165 (bottom) from Table 6 of Dressler \& Gunn (1992). Flux calibration using the spectrophotometric standard G138-31 has been performed. Both spectra have been smoothed with a box car of size 12 A. The galaxy redshifts are determined from several spectral lines that include $\mathrm{Ca}$ II $\mathrm{H}$ and $\mathrm{K}, \mathrm{H} \theta, \mathrm{H} \eta, \mathrm{H} \zeta, \mathrm{H} \epsilon, \mathrm{H} \delta, \mathrm{H} \gamma$, and $\mathrm{H} \beta$. 

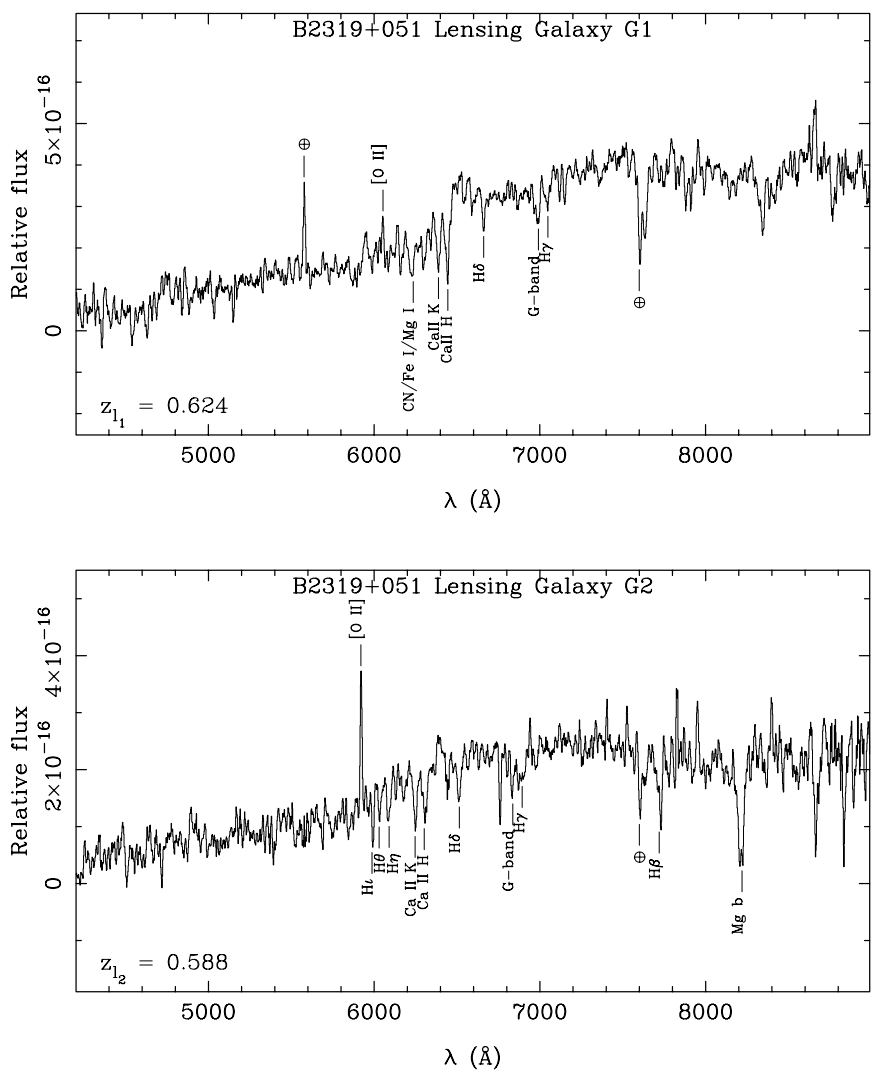

FIG. 6.-LRIS spectra of the lensing galaxies G1 (top) and G2 (bottom) in the CLASS B2319+051 system. Flux calibration using the spectrophotometric standards BD $+33^{\circ} 2641$ for G1 and Feige 110 for G2 has been performed. Each spectrum has been smoothed with a box car of size $12 \AA$. The previously unmeasured redshifts of $z_{l, 1}=0.624$ and $z_{l, 2}=0.588$ are determined from several spectral lines that include [O II], $\mathrm{Ca}$ II $\mathrm{H} \& \mathrm{~K}$, $\mathrm{H} \delta$, and $\mathrm{H} \gamma$.

burst of star formation within the last 1-2 Gyr (see, e.g., $\S 4.2$ ). Such activity is expected as the galaxy appears morphologically irregular with two distinct peaks in the surface brightness profile. This appearance suggests a merger or interaction.

The composite $R$-band image of a $1^{\prime} \times 1^{\prime}$ field centered on B2319+051 is shown in Figure 7. Using the object detection and analysis software SExtractor (Bertin \& Arnouts 1996), we have obtained the magnitude $R=22.2 \pm 0.3$ for the primary lensing galaxy G1 within an aperture the size of the Einstein ring radius (0"68). In addition, the total $R$ magnitudes of $\mathrm{G} 1$ and $\mathrm{G} 2$ are $21.3 \pm 0.3$ and $22.0 \pm 0.3$, respectively. The errors are large because these data were taken in nonphotometric conditions with light-to-moderate cirrus. The total $R-F 160 \mathrm{~W}$ color of G1 is consistent with a nonevolving elliptical at a redshift of $z=0.624$ (Poggianti 1997).

\section{MASS AND LIGHT}

Once the source and lens redshifts of a gravitational lens system are known, the system can be used, in principle, for two distinct purposes. First, it is possible to measure $H_{0}$ by combining the angular diameter distances and a model of the lensing potential to predict the time delays (see, e.g., Refsdal 1964; Blandford \& Narayan 1992; Blandford \& Kundic 1996). The predicted time delay is proportional to the ratio of angular diameter distances, $D \equiv D_{l} D_{s} / D_{l s}$ (where $D_{l}, D_{s}$, and $D_{l s}$ are the angular diameter distances to the lens, to the source, and between the lens and source, respectively). As such, the predicted time delay is also inversely proportional to $h$. Thus, if the background source is variable and the time delays can be measured, the ratio between the observed and predicted time delays will provide a measure of $h$. Unfortunately, a time delay measurement requires long-term radio or optical monitoring and a detection of a relatively strong event (see, e.g., Kundić et al. 1997c; Schechter et al. 1997; Lovell et al. 1998; Biggs et al. 1999; Fassnacht et al. 1999). Consequently, these measurements are difficult to make.

More immediately, gravitational lens systems with measured redshifts can be used to study the properties of massive galaxies at moderate redshifts. Specifically, the size of the image splitting provides a direct estimate of the mass within the Einstein ring of the lens. This mass can be expressed as

$$
M_{\mathrm{E}} \approx 1 \times 10^{12}\left(\frac{D}{1 \mathrm{Gpc}}\right)\left(\frac{\Theta_{\mathrm{E}}}{3^{\prime \prime}}\right)^{2} M_{\odot},
$$

where $\Theta_{\mathrm{E}}$ is the angular radius of the Einstein ring. For the lenses presented in this paper, we find physical Einstein ring radii of $2.6-4.3 h^{-1} \mathrm{kpc}$ and masses of $\sim 1-2 \times 10^{11} h^{-1}$ $M_{\odot}$ (see Table 3).

The mass of the galaxy, combined with its photometric properties, can be used to compute the mass-to-light ratio of the lens. For this calculation, we need to measure the galaxy light within the same aperture as the mass. For both SBS $0909+532$ and HST $1411+5211$, all of the necessary parameters for the mass-to-light ratio calculation have been measured. For the remaining system, B2319+051, we can only provide a reasonable estimate. In the calculations presented below, all of the galaxy magnitudes are given in a Vega-based ("Johnson") magnitude system. In addition, we have converted all observed magnitudes to the rest-frame $B$ band using no-evolution $k$-corrections and rest-frame colors calculated from the spectral energy distribution of a typical elliptical galaxy (Coleman, Wu, \& Weedman 1980). We have ignored the effects of extinction and evolution. While the total extinction is usually modest in early-type lenses $[E(B-V) \leq 0.08 \mathrm{mag}$; Falco et al. 1999], the evolutionary correction is, as expected, an increasing function of redshift, approaching $1 \mathrm{mag}$ at redshifts of $z \sim 0.9$ (Kochanek et al. 2000).

\section{1. $S B S 0909+532$}

The properties of the lensing galaxy in SBS $0909+532$ have been measured by Lehár et al. (2000). They give a total magnitude of $H=16.75 \pm 0.74$, a color of $I-H=2.28 \pm 1.01$ within a 1 ..7 diameter aperture, and an effective radius of $r_{\text {eff }}=11^{\prime \prime} .58 \pm 0.90$. The errors on these parameters are extremely large because the subtraction of the close quasar pair leaves significant residuals in the final image (see Fig. 1 of Lehár et al. 2000). However, we can try to use these values to estimate the light within the Einstein ring radius of 0 "'55. Adopting a de Vaucouleurs law for the galaxy surface brightness profile, we calculate that the magnitude within the Einstein ring radius would be $H=$ $18.3_{-1.0}^{+0.9}$. If we assume that the galaxy color is constant with radius, the $I$ magnitude corresponds to $20.6_{-1.4}^{+1.3}$. Converting this value to an absolute $B$ magnitude, we find $M_{B}=-20.9_{-1.5}^{+1.4}+5 \log h$ and $(M / L)_{B}=4_{-3}^{+11} h(M / L)_{\odot}$. 


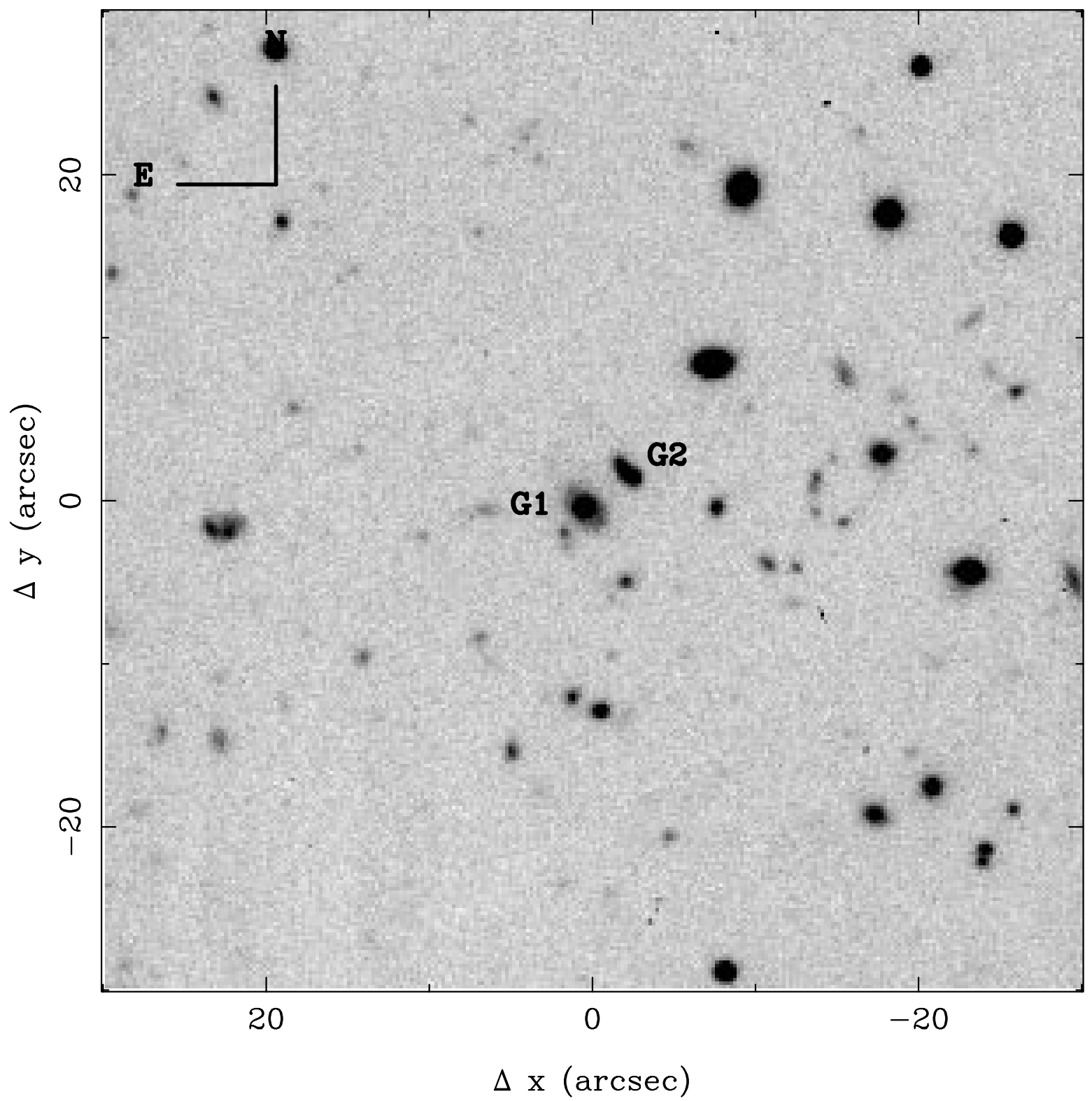

FIG. 7. - Composite $R$-band image centered on the gravitational lens $\mathrm{B} 2319+052$. The field of view is $1^{\prime} \times 1^{\prime}$, and the total exposure time is $1200 \mathrm{~s}$. The lensing galaxies $\mathrm{G} 1$ and $\mathrm{G} 2$ are labeled.

Although this measurement does not place any strong constraints on the $M / L$ of this lensing galaxy, it is consistent with the mass-to-light ratios of other early-type lenses at $z \sim 0.8$. From the review of Keeton et al. (1998), we would expect $(M / L)_{B} \approx 8-16 h(M / L)_{\odot}$. We note that the mass-tolight ratios of high-redshift lensing galaxies are higher (by a factor of $\sim 1.5-2)$ than the $M / L$ ratios of nearby elliptical galaxies within the same physical radius (e.g., Lauer 1985; van der Marel 1991); however, searches for gravitational lenses are biased toward high-mass systems since these systems have a larger cross-section for lensing.

\subsection{HST $1411+5211$}

For HST $1411+5211$, we have obtained the photometry of the lensing galaxy from the processed WFPC2 image of the cluster CL 3C 295 that is given in Smail et al. (1997). We adopt a zero point in the F702W bandpass of $22.38 \pm 0.02$ mag $\mathrm{DN}^{-1} \mathrm{~s}^{-1}$ (Holtzman et al. 1995) and measure an aperture magnitude of $\mathrm{F} 702 \mathrm{~W}=21.23 \pm 0.03$ within the Einstein ring radius of 1 "14. Converting this value to an absolute $B$ magnitude, we find $M_{B}=-18.72 \pm 0.03$ $+5 \log h$ and $(M / L)_{B}=41.3 \pm 1.2 h(M / L)_{\odot}$. This mass-tolight ratio is considerably higher (by a factor of $\sim 5$ ) than the average lensing galaxy at $z \sim 0.4$ (Keeton et al. 1998). The inflated value is the result of cluster-assisted galaxy lensing induced by the cluster CL 3C 295; this cluster is extremely massive with a velocity dispersion of $\sigma=1670$ $\mathrm{km} \mathrm{s}^{-1}$ (Dressler \& Gunn 1992). Such an effect is also seen in the gravitational lens system Q0957 + 561, where the contribution of the $\sigma=730 \mathrm{~km} \mathrm{~s}^{-1}$ cluster (Angonin-Willaime, Soucail, \& Vanderriest 1994; Fischer et al. 1997) results in an unusually high value of $(M / L)_{B} \approx 22 h$ for the central lensing galaxy G1 (Keeton et al. 1998).

\subsection{CLASS B2319+051}

For B2319+051, we have calculated an aperture magnitude of $R=22.2 \pm 0.3$ for the lensing galaxy G1 (see $\S 4.3$ ). 
This magnitude corresponds to $M_{B}=-20.4 \pm 0.3$ $+5 \log h$, or a luminosity of $L_{B}=2.3 \pm 0.6 \times 10^{10} h^{-2} L_{\odot}$. Because the redshift of the background source in this system is not known, we cannot calculate the mass-to-light ratio of the lensing galaxy. However, using the measured luminosity and equation (1), we can represent the $M / L$ ratio of $G 1$ as a function of $D_{s} / D_{l s}$. That is,

$$
\left(\frac{M}{L}\right)_{B} \approx 2.00\left(\frac{D_{s}}{D_{l s}}\right) h\left(\frac{M}{L}\right)_{\odot} .
$$

For reasonable values of the source redshift, i.e., $z_{s}=1-3$, we estimate that $(M / L)_{B}$ will be between $7-3 h(M / L)_{\odot}$. In our chosen cosmology, all other lensing galaxies that have been morphologically classified as early-type galaxies have blue mass-to-light ratios that are greater than $5 h$ (Keeton et al. 1998). In order for the early-type lensing galaxy in B2319+051 to be consistent with the measurements from other lenses, we predict that the source redshift $z_{s}$ will be less than 1.5.

\section{CONCLUSIONS}

As part of a continuing observational program to study gravitational lens systems, we have measured previously unidentified redshifts in three lens systems, SBS 0909+532, HST $1411+5211$, and CLASS B2319+051. The spectral characteristics of the central lensing galaxy in all three systems suggest that each is an early-type galaxy. High angular resolution $H S T$ images confirm that these lenses appear as morphologically normal early-type galaxies (Fischer et al. 1998; Marlow et al. 2000; Lehár et al. 2000). The observations suggest, as previously noted, that the majority of lensing galaxies are early types (see Keeton et al. 1998 and references therein). For the lensing galaxy in HST $1411+5211$, we measure a blue mass-to-light ratio that is a factor of $\sim 5$ larger than the average lensing galaxy at a similar redshift. The presence of the massive cluster CL 3C 295 is responsible for this significantly enhanced ratio.

For the other two systems, we are only able to constrain the mass-to-light ratios. The large observational uncertainties on the luminosity of the lensing galaxy in SBS $0909+532$ allow a wide range in the mass-to-light ratio; however, our measurement is consistent with the observed values in other high-redshift gravitational lenses. Similarly for the primary lensing galaxy in B2319+051, we predict a mass-to-light ratio that is typical of previous lens measurements. Our imaging indicates that both lenses have a few companion galaxies within $200 \mathrm{~h}^{-1} \mathrm{kpc}$ that have magnitudes and/or colors typical of an early-type galaxy at the lens redshift. Consequently, the primary lensing galaxy may be associated with a group of galaxies, as previously observed in the lens systems MG $0751+2716$, PG $1115+080$, and B1422+231 (Kundić et al. 1997a, 1997b; Tonry 1998; Tonry \& Kochanek 1999). We are currently pursuing the group hypothesis for both SBS 0909+532 and B2319+051.

Finally, the expected time delays in all three lens systems are approximately $100 h^{-1}$ days or less (Oscoz et al. 1997; Fischer et al. 1998; Marlow et al. 2000), and at least one source (CLASS B2319+051) shows evidence of variability (Marlow et al. 2000). Therefore, some of these systems may be suitable for measuring $H_{0}$.

We would like to thank the referee, Emilio Falco, for very useful comments on the text. We thank Mark Metzger, Gordon Squires, and Chuck Steidel for helpful discussions and essential material aids to this paper. The W. M. Keck Observatory is operated as a scientific partnership among the California Institute of Technology, the University of California, and the National Aeronautics and Space Administration. It was made possible by the generous financial support of the W. M. Keck Foundation. MERLIN is operated as a national facility by the Nuffield Radio Astronomy Laboratories, University of Manchester, on behalf of the UK Particle Physics and Astronomy Research Council. Support for L. M. L. was provided by NASA through Hubble Fellowship grant HF-01095.01-97A awarded by the Space Telescope Science Institute, which is operated by the Association of Universities for Research in Astronomy, Inc., for NASA under contract NAS 5-26555. This work was partially supported by NSF under grant AST 94-20018.

\section{REFERENCES}

Angonin-Willaime, M.-C., Soucail, G., \& Vanderriest, C. 1994, A\&A, 291, 411

Bertin, E., \& Arnouts, S. 1996, A\&A, 117, 393

Biggs, A. D., Browne, I. W. A., Helbig, P., Koopmans, L. V. E., Wilkinson, P. N., \& Perley, R. A. 1999, MNRAS, 304, 349

Blandford, R. D., \& Kundić, T. 1996, in The Extragalactic Distance Scale, ed. M. Livio (Cambridge: Cambridge Univ. Press), 60

Blandford, R. D., \& Narayan, R. 1992, ARA\&A, 30, 311

Butcher, H., \& Oemler, A. 1978, ApJ, 226, 559

Coleman, G. D., Wu, C. C., \& Weedman, D. W. 1980, ApJS, 43, 393

Dressler, A., \& Gunn, J. E. 1983, ApJ, 270, 7 1992, ApJS, 78, 1

Engels, D., Hagen, H.-J., Cordis, L., Koehler, S., Wisotzki, L., \& Reimers, D. 1998, A\&AS, 128, 507

Falco, E. E., et al. 1999, ApJ, 523, 617

Falco, E. E., Kochanek, C. S., \& Muñoz, J. A. 1998, ApJ, 494, 47

Fassnacht, C. D., et al. 1999, AJ, 117, 658

Fassnacht, C. D., \& Cohen, J. G. 1998, AJ, 115, 377

Fischer, P., Bernstein, G., Rhee, G., \& Tyson, J. A. 1997, AJ, 113, 521

Fischer, P., Schade, D., \& Barrientos, L. F. 1998, ApJ, 503, L127

Helbig, P., Marlow, D., Quast, R., Wilkinson, P. N., Browne, I. W. A., \& Koopmans, L. V. E. 1999, A\&AS, 136, 297

Holtzman, J. A., Burrows, C. J., Casertano, S., Hester, J. J., Trauger, J. T., Watson, A. M., \& Worthey, G. 1995, PASP, 107, 1065

Jackson, N., Helbig, P., Browne, I. W. A., Fassnacht, C. D., Koopmans, L., Marlow, D., \& Wilkinson, P. N. 1998a, A\&A, 334, L33
Jackson, N., et al. 1998b, MNRAS, 296, 483

Keeton, C. R., Kochanek, C. S., \& Falco, E. E. 1998, ApJ, 509, 561

Kennefick, J. D., Djorgovski, S. G., \& de Carvalho, R. R. 1995, AJ, 110, 2553

Kochanek, C. S., et al. 2000, ApJ, submitted (astro-ph/9909018)

Kochanek, C. S., Falco, E. E., \& Schild, R. 1995, ApJ, 452, 109

Kochanek, C. S., Falco, E. E., Schild, R., \& Dobrzycki, A. 1997, ApJ, 479, 678

Koopmans, L. V. E., de Bruyn, A. G., \& Jackson, N. 1998, MNRAS, 295, 534

Koopmans, L. V. E., et al. 1999, MNRAS, 303, 727

Kundić, T., Cohen, J. G., Blandford, R. G., \& Lubin, L. M. 1997a, AJ, 114, 507

Kundić, T., Hogg, D. W., Blandford, R. G., Cohen, J. G., Lubin, L. M., \& Larkin, J. E. 1997b, AJ, 114, 2276

Kundić, T., et al. 1997c, ApJ, 482, 75

Lauer, T. 1985, ApJ, 292, 104

Lehár, J., et al. 2000, ApJ, submitted (astro-ph/9909072)

Leitherer, C. et al. 1996, PASP, 108, 996

Lovell, J. E. J., Jauncey, D. L., Reynolds, J. E., Wieringa, M. H., King, E. A., Tzioumis, A. K., McCulloch, P. M., \& Edwards, P. G. 1998, ApJ, 508, L51

Marlow, D. R., et al. 2000, in preparation

Myers, S. T., et al. 2000, in preparation

Oke, J. B. 1990, AJ, 99, 1621

Oke, J. B., et al. 1995, PASP, 107, 375 
Oke, J. B., \& Korycansky, D. G. 1982, ApJ, 255, 11

Oscoz, A., Serra-Ricart, M., Mediavilla, E., Buitrago, J., \& Goicoecheo, L. J. 1997, ApJ, 491, L7

Poggianti, B. M. 1997, A\&AS, 112, 399

Refsdal, S. 1964, MNRAS, 128, 307

Schechter, P. L., et al. 1997, ApJ, 475, 85

Smail, I., Dressler, A., Couch, W. J., Ellis, R. E., Oemler, A., Butcher, H. R., \& Sharples, R. M. 1997, ApJS, 110, 213

Steidel, C. C., Giavalisco, M., Dickinson, M., \& Adelberger, K. L. 1996a, AJ, 112,352

Steidel, C. C., Giavalisco, M., Pettini, M., Dickinson, M., \& Adelberger, K. L. 1996b, ApJ, 462, L17
Stepanyan, D. A., Lipovetskii, V. A., Chavushyan, V. O., Erastova, L. K., \& Shapovalova, A. I. 1991, Astrofizika, 34, 1

Sykes, C. M., et al. 1998, MNRAS, 301, 310

Thimm, G. J., Roser, H. J., Hippelein, H., \& Meisenheimer, K. 1994, A\&A, 285,785

Tonry, J. L. 1998, AJ, 115, 1

Tonry, J. L., \& Kochanek, C. S. 1999, AJ, 117, 2034

van der Marel, R. P. 1991, MNRAS, 253, 710

Xanthopoulos, E., et al. 1998, MNRAS, 300, 649

Zabludoff, A. I., Zaritsky, D., Lin, H., Tucker, D., Hashimoto, Y., Shectman, S. A., Oemler, A., \& Kirshner, R. P. 1996, ApJ, 466, 104 